\title{
DETERMINATION OF FABRIC SURFACE RESISTANCE BY VAN DER PAUW METHOD IN CASE OF CONTACTS DISTANT FROM THE SAMPLE EDGE
}

\author{
Magdalena Tokarska \\ Department of Architecture of Textiles, Lodz University of Technology, 116 Zeromskiego St., 90-924 Lodz Poland \\ E-mail: magdalena.tokarska@p.lodz.pl
}

\begin{abstract}
:
The van der Pauw method can be used to determine the electroconductive properties of textile materials. However, the sample surface resistance can be determined provided that the sample has characteristics typical for the van der Pauw structure. In the paper, a method of evaluating the sample structure is shown. The selected electroconductive woven fabrics are used as an example of van der Pauw structure. An analysis of impact of electrodes placement on the resistance measurements was conducted. Knowing how the resistance of the sample varies with the electrodes distance from the edge, the samples' surface resistances were calculated in cases when the electrodes are placed at the sample edge. An uncertainty analysis of the samples' surface resistances was conducted based on the Monte Carlo method.
\end{abstract}

\section{Keywords:}

Electroconductive properties, woven fabric, van der Pauw structure, van der Pauw method, surface resistance, electrodes placement, Monte Carlo method

\section{Introduction}

The van der Pauw method was developed to determine the properties of thin semiconductors [1,2]. This method is currently used to evaluate the conductivity of thin layers deposited on different substrates [3-6] or thin electroconductive materials [7-10]. The van der Pauw method can be applied to small samples contrary to the four electrode method [11] where electrodes are located collinearly on the tested material surface. The surface resistance can be determined using the van der Pauw equation [1] provided that the electroconductive sample has the structure typical for that used by van der Pauw. An evaluation of the fibrous structure from this point of view is difficult and requires a detailed analysis of its structure. The complexity of a textile sample, for example woven fabric, shows the number of different parameters describing the structure $[12,13]$. In order to verify whether the conditions described in the van der Pauw method are represented in the woven fabric structure, a special criterion was developed [7]. The criterion is useful in selecting appropriate fabric sample for testing.

The van der Pauw method requires that the point contacts used for the resistance measurements be placed at the edges of the sample. The effect of electrode placement on resistance measurement was investigated $[8,10,14]$. In the case of the woven fabrics, the contacts should not be too close to the boundaries of the sample because errors can be introduced $[3,15]$. In this paper, a solution for the problem is proposed. Knowing how the resistance of the sample varies with the electrodes distance from the edges, the sample surface resistance can be calculated in cases when the electrodes placement is consistent with the van der Pauw notion.

\section{Materials}

Two electroconductive woven fabrics were selected for testing. The first fabric, labelled as $\mathrm{S} 1$, is an antioxidant silver fibre shielding rip-stop fabric. The rip-stop fabric is resistant to tearing and ripping. The second sample, signed as S2, is a silver fibre shielding canvas fabric. The canvas is a tight fabric and very durable. The chosen fabrics parameters and their standard deviation are juxtaposed in Table 1.

The samples with a measuring area in the shape of a $70 \mathrm{~mm}$ side square were prepared from the electroconductive woven

Table 1. Parameters of the electroconductive woven fabrics.

\begin{tabular}{|c|c|c|c|c|c|}
\hline \multirow{2}{*}{ Sample } & Thickness & $\begin{array}{c}\text { Standard } \\
\text { deviation of } \\
\text { thickness }\end{array}$ & Areal density & $\begin{array}{c}\text { Standard } \\
\text { deviation of areal } \\
\text { density }\end{array}$ & $\begin{array}{c}\text { Volumetric } \\
\text { density }\end{array}$ \\
\cline { 2 - 6 } & $\mathbf{m m}$ & $\mathbf{m m}$ & $\mathbf{g} / \mathbf{m}^{2}$ & $\mathbf{g} / \mathbf{m}^{2}$ & $\mathbf{k g} / \mathbf{m}^{3}$ \\
\hline$S 1$ & 0.15 & $1.4 \times 10^{-3}$ & 76 & 0.12 & 507 \\
\hline$S 2$ & 0.35 & $1.4 \times 10^{-3}$ & 149 & 0.31 & 426 \\
\hline
\end{tabular}


fabrics. The direction of cut lines was perpendicular to warp and weft yarns.

The structure of the selected fabrics was analysed in detail. It was examined if the fabrics possess characteristics typical for the van der Pauw structure [1]. A special criterion was developed by Tokarska [7] based on $[11,12,16]$ to assess the following characteristics:

- geometry of the sample (A),

- compactness of the structure (B),

- $\quad$ homogeneity of the structure $(C)$,

- $\quad$ isotropy of the sample (D).

Accordingly, the measures given below were used.

A) The measures of the sample geometry:

$$
h \leq 1 \mathrm{~mm}
$$

where $h$ is the sample thickness;

$$
V_{h}=\frac{h_{a v}}{s_{h}} 100 \% \leq 1 \%
$$

where $h_{a v}$ is the average sample thickness, $s_{h}$ is the standard deviation and $V_{h}$ is the coefficient of variation of the sample thickness;

$$
G=\frac{L}{h} \geq 15
$$

where $L$ is the peripheral length of the sample.

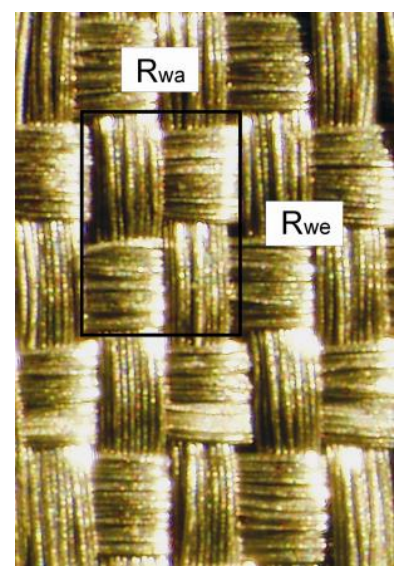

Sample S1
B) The measure of the structure compactness:

$$
C=\frac{A_{w e} d_{w a}+A_{w a} d_{w e}-d_{w e} d_{w a}}{A_{w a} A_{w e}} 100 \geq 95
$$

where $A_{w a}$ is the spacing of the warp yarns, $A_{\text {we }}$ is the spacing of the weft yarns, $d_{w a}$ is the diameter of warp yarns and $d_{w e}$ is the diameter of weft yarns.

C) The measure of the structure homogeneity:

$$
H=\frac{R_{w a} A_{w a} R_{w e} A_{w e}}{S} \leq 0.001
$$

where $R_{w a}$ and $R_{w e}$ are the warp and weft repeats, respectively, and $S$ is the surface of the tested sample.

D) The measure of the sample isotropy:

$$
I z=\frac{\min \left\{R \alpha_{1}, R \alpha_{2}, \ldots, R \alpha_{n}\right\}}{\max \left\{R \alpha_{1}, R \alpha_{2}, \ldots, R \alpha_{n}\right\}} \geq 0.95
$$

where $R \alpha_{1}, R \alpha_{2}, \ldots, R \alpha_{n}$ are resistances measured in the selected $n$ directions using four electrodes located collinearly on the sample [11].

Analysis of the fabrics structure was conducted based on microscopic images of the samples taken with Olympus microscope. The images were captured at $7.5^{\prime}$ magnification (Figure 1).

The designated parameters are juxtaposed in Table 2.

Figure 1. Microscopic images of the samples.

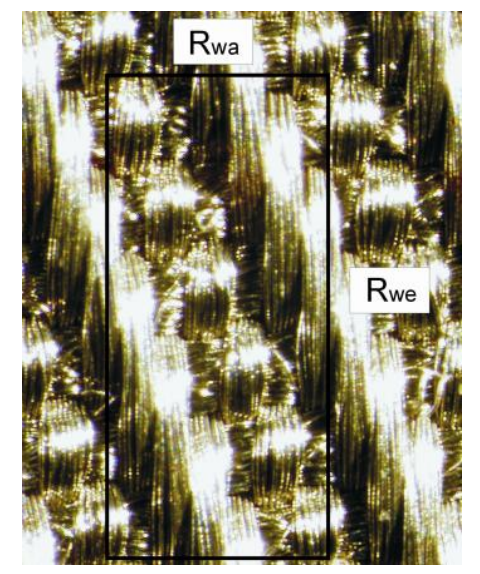

Sample S2

Table 2. Parameters of the fabrics for the analysis of the fabric structure.

\begin{tabular}{|c|c|c|c|c|c|c|c|c|c|}
\hline Sample & $\boldsymbol{L}$ & $\boldsymbol{R}_{\text {wa }}$ & $\boldsymbol{d}_{\text {wa }}$ & $\boldsymbol{A}_{\text {wa }}$ & $\boldsymbol{R}_{\text {we }}$ & $\boldsymbol{d}_{\text {we }}$ & $\boldsymbol{A}_{\text {we }}$ & $\boldsymbol{N}_{\text {wa }}$ & $\boldsymbol{N}_{\text {we }}$ \\
\hline- & $\mathrm{mm}$ & units & $\mathrm{mm}$ & $\mathrm{mm}$ & units & $\mathrm{mm}$ & $\mathrm{mm}$ & $\begin{array}{c}\text { yarns per } \\
1 \mathrm{~cm}\end{array}$ & $\begin{array}{c}\text { yarns per } \\
1 \mathrm{~cm}\end{array}$ \\
\hline $\mathrm{S} 1$ & 280 & 2 & 0.21 & 0.24 & 2 & 0.28 & 0.32 & 41 & 31 \\
\hline $\mathrm{S} 2$ & 280 & 6 & 0.14 & 0.14 & 6 & 0.27 & 0.30 & 71 & 33 \\
\hline
\end{tabular}


The designated parameters, $A_{\text {wa }}$ and $A_{\text {we }}$, allowed calculating the number of warp yarns, $N_{\text {wa }}$, and the number of weft yarns, $N_{\text {we }}$, per $1 \mathrm{~cm}$ [12]. The received results are summarised in Table 2.

The characteristics typical for a van der Pauw structure have been determined by the implemented measures, i.e.: $h$ calculated from formula (1), $V h$ from (2), $G$ from (3), C from (4) and $H$ from (5). In order to determine $I z$ from the formula, four electrodes located collinearly on the sample were used [11]. The cylindrical brass silver-plated electrodes of the contact diameter equal to $2 \mathrm{~mm}$ were used. The electrodes placement on the fabric sample is presented in Figure 2.

The method can be applied when the ratio $a / D$ is greater than 2 , where $a$ is the distance from the edge of the sample and $D$ is the distance between electrodes [17], which is shown in Figure 2.

The resistances $R \alpha_{1}, R \alpha_{2}, R \alpha_{3}$ and $R \alpha_{4}$ were measured in the selected $n=4$ directions (Figure 2) by injecting current on two

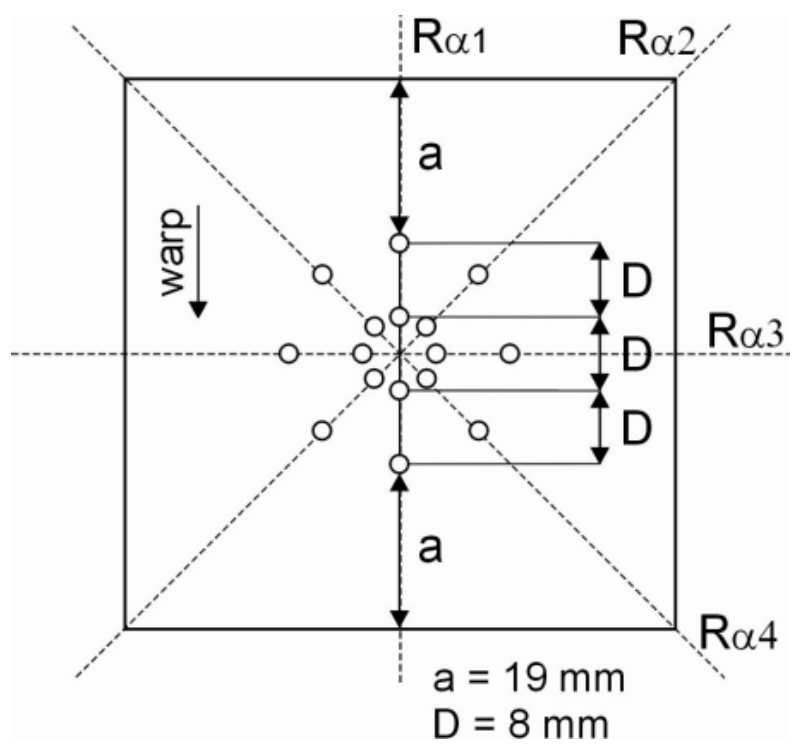

Figure 2. The electrodes placement on the fabric sample - four electrode method. external contacts and by measuring the voltage drop on the two internal ones. The received results are presented in Table 3.

All the received results are summarised in Table 4 .

It was finally confirmed that the selected electroconductive woven fabrics had all the characteristics typical for a van der Pauw structure.

\section{Methods}

The van der Pauw method was used for the determination of surface resistance of the woven fabrics. The technique requires four contacts as small as possible placed on the sample edges. Cylindrical brass silver-plated electrodes of the contact diameter equal to $2 \mathrm{~mm}$ were used. The electrodes have a comparatively small contact area with the sample relative to the sample surface. The contact area of the electrode was selected so that it covers the repeat of the tested fabric. It is very important to ensure that all yarns are contacted during measurements.

A surface resistance $R_{\mathrm{s}}$ of the sample having a structure typical for that used by van der Pauw can be determined from equation [1]:

$$
\exp \left(-\pi \frac{R_{v}}{R_{s}}\right)+\exp \left(-\pi \frac{R_{h}}{R_{s}}\right)-1=0
$$

where $R_{\mathrm{v}}$ is the vertical resistance and $R_{\mathrm{h}}$ is the horizontal resistance.

The surface resistance is expressed in $\Omega / \square(\Omega$ per square). The resistance is numerically equal to the resistance of a square piece of the sample.

For vertical resistance $R_{\mathrm{v}}$ measurement (for instance, $R_{\mathrm{A}-\mathrm{D}, \mathrm{B}-\mathrm{C}}$ ), a voltage is applied to flow current $I_{\mathrm{A}-\mathrm{D}}$ along one side of the square sample and the voltage drop $V_{\mathrm{B}-\mathrm{C}}$ along the opposite side is measured (Figure 3). For horizontal resistance $R_{\mathrm{h}}$ measurement (for instance, $R_{\mathrm{A}-\mathrm{B}, \mathrm{D}-\mathrm{C}}$ ), a voltage is applied to flow current $I_{\mathrm{A}-\mathrm{B}}$ along one side of the square sample and the voltage drop $V_{\mathrm{D}-\mathrm{C}}$ along the opposite side is measured (Figure 3).

Table 3. Values of resistances measured in the selected directions.

\begin{tabular}{|c|c|c|c|c|c|}
\hline \multicolumn{2}{|c|}{ Sample } & $\mathbf{R a}_{\mathbf{1}}$ & $\mathbf{R a}_{\mathbf{2}}$ & $\mathbf{R \alpha}_{\mathbf{3}}$ & $\mathbf{R \alpha}_{\mathbf{4}}$ \\
\hline \multirow{2}{*}{ S1 } & Resistance $(\Omega)$ & 0.0377 & 0.0374 & 0.0372 & 0.0371 \\
\cline { 2 - 6 } & Standard deviation $(\Omega)$ & $5.6 \times 10^{-4}$ & $1.7 \times 10^{-3}$ & $9.4 \times 10^{-4}$ & $1.3 \times 10^{-3}$ \\
\hline \multirow{2}{*}{ S2 } & Resistance $(\Omega)$ & 0.0248 & 0.0255 & 0.0257 & 0.0254 \\
\cline { 2 - 7 } & Standard deviation $(\Omega)$ & $1.0 \times 10^{-3}$ & $4.3 \times 10^{-4}$ & $1.7 \times 10^{-4}$ & $4.9 \times 10^{-4}$ \\
\hline
\end{tabular}

Table 4. Measures of the characteristics typical for the van der Pauw structure.

\begin{tabular}{|c|c|c|c|c|c|c|}
\hline Sample & $\mathbf{h}$ & $\mathbf{V}_{\mathbf{h}}$ & $\mathbf{G}$ & $\mathbf{C}$ & $\mathbf{H}$ & $\mathbf{I z}$ \\
\hline- & $\mathrm{mm}$ & $\%$ & - & - & - & - \\
\hline $\mathrm{S} 1$ & 0.15 & 0.9 & 1867 & 98 & 0.0001 & 0.98 \\
\hline $\mathrm{S} 2$ & 0.35 & 0.4 & 800 & 100 & 0.0003 & 0.96 \\
\hline
\end{tabular}




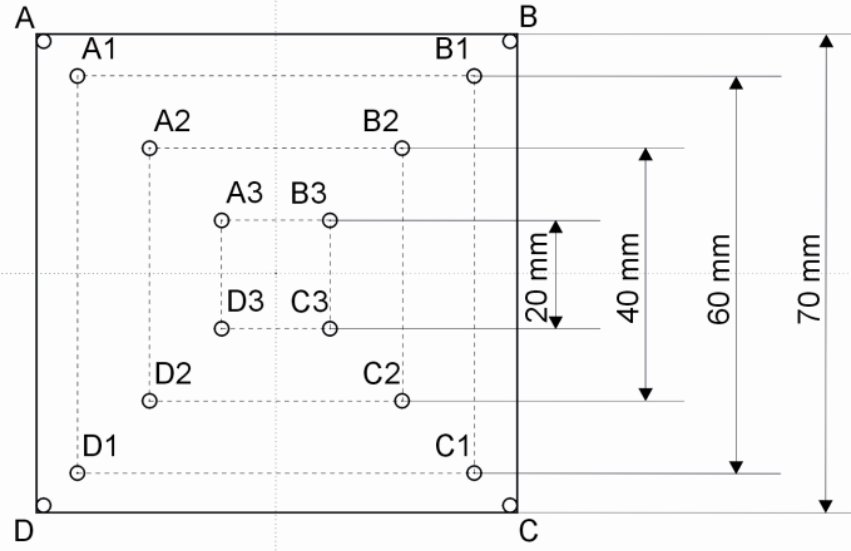

Figure 3. The electrodes placement on the fabric sample - van der Pauw method.

In order to improve the accuracy of the vertical and horizontal resistance values, determination of eight resistances is recommended [18]. The average vertical resistance is then calculated from the formula:

$$
R_{v}=\frac{R_{A-D, B-C}+R_{D-A, C-B}+R_{B-C, A-D}+R_{C-B, D-A}}{4}
$$

The average horizontal resistance is then calculated from the formula:

$$
R_{h}=\frac{R_{A-B, D-C}+R_{B-A, C-D}+R_{D-C, A-B}+R_{C-D, B-A}}{4} \text { (9) }
$$

It has been observed that electrodes should not be too close to the edge of the sample that the error will not be introduced $[3,15]$. To avoid this, additional measurements need to be performed. A table with four holes drilled was prepared allowing the electrodes to fall freely under their weight onto the textile material [15]. This table allowed electrodes to be arranged in the shape of a square with side of $20 \mathrm{~mm}, 40 \mathrm{~mm}$ and 60 $\mathrm{mm}$ (Figure 3). The following resistances can be determined:
$R_{\mathrm{Ai}-\mathrm{Di}, \mathrm{Bi}-\mathrm{Ci}}, \quad R_{\mathrm{Di}-\mathrm{Ai}, \mathrm{Ci}-\mathrm{Bi}}, \quad R_{\mathrm{Bi}-\mathrm{Ci}, \mathrm{Ai}-\mathrm{Di}}, \quad R_{\mathrm{Ci}-\mathrm{Bi}, \mathrm{Di}-\mathrm{Ai}}$ and $R_{\mathrm{Ai}-\mathrm{Bi}, \mathrm{Di}-\mathrm{Ci}}, \quad R_{\mathrm{Bi}-\mathrm{Ai}, \mathrm{Ci}-\mathrm{Di}}$, $R_{\mathrm{Di}-\mathrm{C}, \mathrm{Ai}-\mathrm{Bi}}$ and $R_{\mathrm{Ci}-\mathrm{Di} \mathrm{Bi}-\mathrm{Ai}}$, where $i=1,2,3$, with the use of the table. Next, the vertical and horizontal resistances can be calculated i.e. $R_{\mathrm{vi}}(8)$ and $R_{\mathrm{hi}}(9)$ for $i=1,2,3$. The received values allow determining the relationships:

$$
\begin{aligned}
& R_{h}=f_{h}(l) \\
& R_{v}=f_{v}(l)
\end{aligned}
$$

where $/$ is the electrode distance from the sample edge.

In the presented studies, the following distances are taken into consideration: $I_{1}=\left|\overrightarrow{B B_{1}}\right|, I_{2}=\left|\overrightarrow{B B_{2}}\right|$ and $I_{3}=\left|\overrightarrow{B B_{3}}\right|$ resulting from the electrode placement (Figure 3 ). Assuming $I=0$, in formulae (10) and (11), it is possible to obtain values of the horizontal and vertical resistances respectively in the case when the electrodes are placed at the sample edges. Then, the sample surface resistance can be calculated.

\section{Results and discussion}

The samples were stored for $24 \mathrm{~h}$ in the standard atmosphere conditions $\left(20^{\circ} \mathrm{C}\right.$ and $\left.65 \% \mathrm{RH}\right)$ [19]. The measurements were performed in the same conditions. A DC power supply Agilent E3644A was used as an ammeter. The resolution of the ammeter was $0.001 \mathrm{~A}$. A multimeter Agilent 34410A was used as a voltmeter. The resolution of the voltmeter was $0.001 \mathrm{mV}$. A current $I$ of an intensity of 0.040 A was applied. The voltage drop $\checkmark$ was recorded after $60 \mathrm{~s}$. The resistance value was calculated from the Ohm's law. The measurements were repeated three times. The vertical and horizontal resistances were calculated according to the formulae (8) and (9). The received results

\begin{tabular}{|c|c|c|c|c|c|c|c|c|c|}
\hline \multirow{2}{*}{ Sample } & \multirow{2}{*}{ Distance } & \multirow{2}{*}{ Resistance } & Estimate & $\mathrm{u}_{\mathrm{B}}(\mathrm{I})$ & $\partial \mathrm{R} / \partial \mathrm{I}$ & $u_{A}(V)$ & $u_{B}(V)$ & $\partial \mathbf{R} / \partial \mathbf{V}$ & $\mathbf{U}$ \\
\hline & & & $\mathbf{\Omega}$ & A & V/A ${ }^{2}$ & $\mathbf{V}$ & $\mathbf{V}$ & $1 / A$ & $\mathbf{\Omega}$ \\
\hline \multirow{6}{*}{$\mathrm{S} 1$} & \multirow{2}{*}{$I_{1}=7.07 \mathrm{~mm}$} & $R v_{1}$ & 0.0126 & $5.8 \cdot 10^{-4}$ & 0.314 & $5.7 \cdot 10^{-6}$ & $5.8 \cdot 10^{-7}$ & 25 & 0.0005 \\
\hline & & $R h_{1}$ & 0.0159 & $5.8 \cdot 10^{-4}$ & 0.397 & $6.2 \cdot 10^{-6}$ & $5.8 \cdot 10^{-7}$ & 25 & 0.0006 \\
\hline & \multirow{2}{*}{$I_{2}=21.21 \mathrm{~mm}$} & $R v_{2}$ & 0.0108 & $5.8 \cdot 10^{-4}$ & 0.270 & $5.4 \cdot 10^{-6}$ & $5.8 \cdot 10^{-7}$ & 25 & 0.0004 \\
\hline & & $R h_{2}$ & 0.0151 & $5.8 \cdot 10^{-4}$ & 0.379 & $5.4 \cdot 10^{-6}$ & $5.8 \cdot 10^{-7}$ & 25 & 0.0005 \\
\hline & \multirow{2}{*}{$I_{3}=35.36 \mathrm{~mm}$} & $R v_{3}$ & 0.0088 & $5.8 \cdot 10^{-4}$ & 0.219 & $1.0 \cdot 10^{-5}$ & $5.8 \cdot 10^{-7}$ & 25 & 0.0006 \\
\hline & & $R h_{3}$ & 0.0131 & $5.8 \cdot 10^{-4}$ & 0.327 & $6.9 \cdot 10^{-6}$ & $5.8 \cdot 10^{-7}$ & 25 & 0.0005 \\
\hline \multirow{6}{*}{$\mathrm{S} 2$} & \multirow{2}{*}{$I_{1}=7.07 \mathrm{~mm}$} & $R v_{1}$ & 0.0218 & $5.8 \cdot 10^{-4}$ & 0.546 & $1.2 \cdot 10^{-5}$ & $5.8 \cdot 10^{-7}$ & 25 & 0.0009 \\
\hline & & $R h_{1}$ & 0.0235 & $5.8 \cdot 10^{-4}$ & 0.587 & $1.6 \cdot 10^{-5}$ & $5.8 \cdot 10^{-7}$ & 25 & 0.0010 \\
\hline & \multirow{2}{*}{$I_{2}=21.21 \mathrm{~mm}$} & $R v_{2}$ & 0.0182 & $5.8 \cdot 10^{-4}$ & 0.454 & $8.4 \cdot 10^{-6}$ & $5.8 \cdot 10^{-7}$ & 25 & 0.0007 \\
\hline & & $R h_{2}$ & 0.0184 & $5.8 \cdot 10^{-4}$ & 0.461 & $1.1 \cdot 10^{-5}$ & $5.8 \cdot 10^{-7}$ & 25 & 0.0008 \\
\hline & \multirow{2}{*}{$I_{3}=35.36 \mathrm{~mm}$} & $R v_{3}$ & 0.0119 & $5.8 \cdot 10^{-4}$ & 0.297 & $1.1 \cdot 10^{-5}$ & $5.8 \cdot 10^{-7}$ & 25 & 0.0006 \\
\hline & & $R h_{3}$ & 0.0173 & $5.8 \cdot 10^{-4}$ & 0.432 & $1.1 \cdot 10^{-5}$ & $5.8 \cdot 10^{-7}$ & 25 & 0.0007 \\
\hline
\end{tabular}
and the uncertainty budget determined in accordance with the Guide [20] are summarised in Table 5.

Table 5. Measurement results of vertical and horizontal resistances. 
Type A uncertainty, $u_{A}(V)$, was received for 132 degrees of freedom. Calculating the Type B standard uncertainties, $u_{\mathrm{B}}(l)$ and $u_{\mathrm{B}}(V)$, the rectangular distribution of mezurand values was assumed. The partial derivatives $\partial R / \partial I$ and $\partial R / \partial V$ are the sensitivity coefficients. The expanded uncertainty $U$ was obtained taking a coverage factor equal to $k_{p}=2$.

An analysis of impact of electrodes placement on the resistance measurements was conducted with the use of the KruskalWallis rank sum test [21]. The test is used to verify the hypothesis that $k$ independent samples (in the presented research $k=3$ ) come from the same population. It was found that the manner of the electrodes placement on the samples' surface significantly influences the samples' resistance measurement results at a significance level of 0.05 . The received results indicate that the relationships (10) and (11) are possible to find. For this purpose, non-linear regression analysis was used [22]. The following general form of the regression function was assumed:

$$
R(l)=b_{0}+b_{1} l+b_{2} l^{2}
$$

where $I$ is the electrode distance (expressed in $\mathrm{mm}$ ) from the sample edge and $b_{0}$ (expressed in $\Omega$ ), $b_{1}$ (expressed in $\Omega / \mathrm{mm}$ ) and $b_{2}$ (expressed in $\Omega / \mathrm{mm}^{2}$ ) are the polynomial coefficients.

The following functions were received for sample S1:

$$
\begin{aligned}
& R_{h}(l)=0.0136-0.00013 l \pm 0.0007 \\
& R_{v}(l)=0.0161-0.000002 l^{2} \pm 0.0005
\end{aligned}
$$

The following functions were received for sample S2:

$$
\begin{array}{r}
R_{h}(l)=0.0221-0.000008 l^{2} \pm 0.0009 \\
R_{v}(l)=0.0274-0.00063 l+0.000010 l^{2} \pm 0.0011
\end{array}
$$

The coefficients of the functions (13)-(16) are significant and the functions are adequate at a significance level equal to
0.05 . The graphical form of the received dependences, $R(I)$, is presented in Figure 4.

Assuming $I=0.00 \mathrm{~mm}$ in obtained relationships (13)-(16), the vertical $R_{\mathrm{v}}$ and horizontal $R_{\mathrm{h}}$ resistances values were calculated corresponding to a situation in which the electrodes are placed at the edge of the sample. Next, the surface resistances $R_{\mathrm{s}}$ of the woven fabric samples possessing characteristics typical for the van der Pauw structure were received. The results are juxtaposed in Table 6.

Uncertainty analysis of surface resistance measurements were conducted according to the procedure developed by Tokarska [7] based on the Monte Carlo method [23]. The estimate of the surface resistance, the standard uncertainty associated with the estimate and the probabilistically symmetric 95\% coverage interval defined by the quantiles 0.025 and 0.975 are summarised in Table 7.

Table 6. Resistances obtained for the tested fabrics.

\begin{tabular}{|c|c|c|c|}
\hline \multirow{2}{*}{ Sample } & $\mathbf{R}_{\mathbf{v}}$ & $\mathbf{R}_{\mathbf{h}}$ & $\mathbf{R}_{\mathbf{s}}$ \\
\cline { 2 - 4 } & $\boldsymbol{\Omega}$ & $\boldsymbol{\Omega}$ & $\boldsymbol{\Omega} / \square$ \\
\hline S1 & $0.0136 \pm 0.0007$ & $0.0161 \pm 0.0005$ & 0.0671 \\
\hline S2 & $0.0221 \pm 0.0009$ & $0.0274 \pm 0.0011$ & 0.1117 \\
\hline
\end{tabular}

Table 7. Results of the evaluation of the uncertainty of samples' surface resistance measurements.

\begin{tabular}{|c|c|c|c|}
\hline \multirow{2}{*}{ Sample } & $\begin{array}{c}\text { Estimate } \\
\text { of } \mathbf{R}_{\mathbf{s}}\end{array}$ & $\begin{array}{c}\text { Standard } \\
\text { deviation }\end{array}$ & Coverage interval \\
\cline { 2 - 4 } & $\Omega / \square$ & $\Omega / \square$ & $\Omega / \square$ \\
\hline $\mathrm{S} 1$ & 0.0671 & 0.0012 & {$[0.0650,0.0693]$} \\
\hline $\mathrm{S} 2$ & 0.1117 & 0.0018 & {$[0.1082,0.1152]$} \\
\hline
\end{tabular}

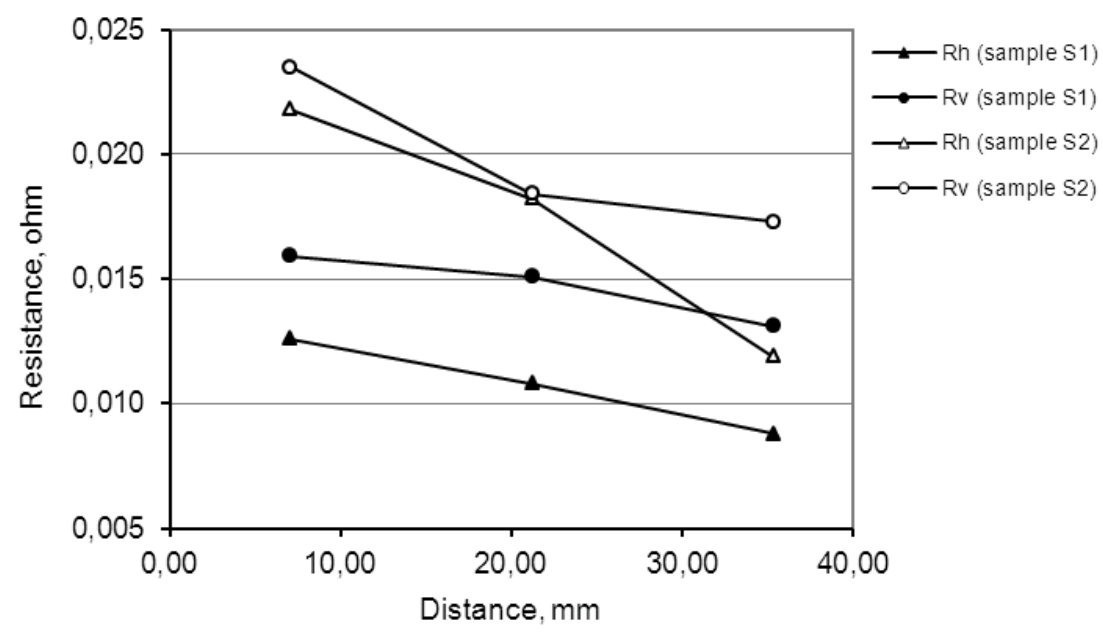

Figure 4. Characteristics received for samples S1 and S2. 


\section{Conclusions}

Results of the study show that the van der Pauw method can be used for the determination of the surface resistance property associated with the surface of the woven fabric sample. However, the electroconductive textile sample should have a structure typical to that used by van der Pauw.

The conducted analysis shows that the electrodes placement on the sample surface influences the resistance measurements. Moving the electrodes towards the centre of the sample decreases the resistance. This is due to the fact that the current flow in the fibrous structure is inhomogeneous. Therefore, determination of the vertical and horizontal resistance values requires the identification of additional eight resistances. The electrodes should not be too close to the edge of the sample that the error will not be introduced. That is why the effect of the electrode placement on the sample resistance is analysed. Knowing how the resistance of the sample varies with the electrodes distance from the edge, the vertical and horizontal resistances can be calculated in cases when the electrodes placement is consistent with the van der Pauw notion. After determining values of the resistances, the sample surface resistance is calculated.

The surface resistance of the sample S1 was received and it is $0.0671 \Omega / \square$. The standard uncertainty associated with the resistance estimate is $0.0012 \Omega / \square$ and the $95 \%$ coverage interval is $[0.0650,0.0693] \Omega / \square$. Surface resistance of the sample S2 was received and it is $0.1117 \Omega / \square$. The standard uncertainty associated with the resistance estimate is $0.0018 \Omega / \square$ and the $95 \%$ coverage interval is $[0.1082$, $0.1152] \Omega / \square$.

\section{References}

[1] Van der Pauw, L.J.: A method of measuring specific resistivity and Hall effect of discs of arbitrary shape. Philips Research Reports 13 (1958), p.1-9

[2] Van der Pauw, L.J.: A method of measuring resistivity and Hall coefficient on lamellae of arbitrary shape. Philips Technical Review 20 (1958/59), p.220-224

[3] Kazani, I.: Study of screen-printed electroconductive textile materials. Doctoral Thesis. Faculty of Engineering and Architecture, Ghent University 2012

[4] Kazani, I., Hertleer, C., De Mey, G., Schwarz, A., Guxho, G., Van Langenhove, L.: Electrical conductive textiles obtained by screen printing. Fibres and Textiles in Eastern Europe 20 (2012), p.57-63

[5] Náhlík, J., Kašpárková, I., Fitl, P.: Influence of non-ideal circumferential contacts on errors in the measurements of the resistivity of layers using the van der Pauw method. Measurement 46 (2013), p.887-892

[6] Kazani, I., De Mey, G., Hertleer, C., Banaszczyk, J., Schwarz, A., Guxho, G., Van Langenhove, L.: Van der Pauw method for measuring resistivities of anisotropic layers printed on textile substrates. Textile Research Journal 81 (2011), p.2117-2124

[7] Tokarska, M.: Evaluation of measurement uncertainty of fabric surface resistance implied by the Van der Pauw equation. IEEE Transactions on Instrumentation and Measurement (2013) DOI: 10.1109/TIM.2013.2289695

[8] Tokarska, M.: Measuring resistance of textile materials based on Van der Pauw method. Indian Journal of Fibre and Textile Research 38 (2013), p.198-201

[9] Kasl, C., Hoch, M.J.R.: Effects of sample thickness on the van der Pauw technique for resistivity measurements. Review of Scientific Instruments 76 (2005), p.033907-1033907-4

[10] Wu, B., Huang, X., Han, Y., Gao, Ch., Peng, G., Liu, C, Wang, Y., Cui, X., Zou, G.: Finite element analysis of the effect of electrodes placement on accurate resistivity measurement in a diamond anvil cell with van der Pauw technique. Journal of Applied Physics 107 (2010), p.104903-1-104903-4

[11] Wenner, F.: A method of measuring earth resistivity. Bulletin of the Bureau of Standards 12 (1916), p.469-478

[12] Szosland, J.: Struktury tkaninowe (in Polish). PAN Lodz 2007

[13] Szosland, J.: Modelling the structural barrier ability of woven fabrics. Autex Research Journal 3 (2003), p.102-110

[14] Lim, S.H.N., McKenzie, D.R., Bilek, M.M.M.: Van der Pauw method for measuring resistivity of a plane sample with distant boundaries. Review of Scientific Instruments 80 (2009), p.075109-1-075109-4

[15] Tokarska, M., Frydrysiak, M., Zięba, J.: Electrical properties of flat textile material as inhomegeneous and anisotropic structure. Journal of Materials Science - Materials in Electronics (2013) DOI: 10.1007/s10854-013-1524-4

[16] ASTM F76-08. Standard Test Methods for Measuring Resistivity and Hall Coefficient and Determining Hall Mobility in Single-Crystal Semiconductors

[17]Deen, M.J., Pascal, F.: Electrical characterizations of semiconductor materials and devices - review. Journal of Materials Science - Materials in Electronics 17 (2006), p. $549-575$

[18] Džakula, R., Savić, S., Stojanović, G.: Investigation of electrical characteristics of different ceramic samples using Hall effect measurement. Processing and Application of Ceramics 2 (2008), p.33-37

[19]ISO 139:2005. Textiles - Standard atmospheres for conditioning and testing

[20] Evaluation of measurement data - Guide to the expression of uncertainty in measurement, JCGM (2008)

[21] Corder, G.W., Foreman, D.I.: Nonparametric statistics for non-statisticians: A step-by-step approach. John Wiley \& Sons 2009

[22] Seber, G.A.F., Wild, C.J.: Nonlinear regression. John Wiley \& Sons 2003

[23] Evaluation of measurement data - Supplement 1 to the "Guide to the expression of uncertainty in measurement" - Propagation of distributions using a Monte Carlo method, JCGM (2008) 